\title{
Research on Wavelet Transform Method Based on SURF Threshold Improved Mallat
}

\author{
Yuan Jiao ${ }^{1, *}$ and Binwen Huang ${ }^{2}$ \\ ${ }^{1}$ Information Technology Department, Hainan Medical University, Haikou, Hainan, 571199, China \\ ${ }^{2}$ Information Technology Department, Hainan Vocational College of Political Science and Law, Haikou, Hainan, \\ 571100, China
}

\begin{abstract}
In this paper, watant role in the signal processing. Analyzing the limitations of Fourier transform in the signal processing, this article reflects the superiority of the wavelet transform, analyzes the principle of Mallat wavelet transform, builds decomposition and reconstruction model of Mallat wavelet, and introduces the SURF threshold to improve Mallat transform method. Finally, comparing the denoising results of single Mallat and improved Mallat, the paper verifies that the improved Mallat method is feasible.
\end{abstract}

Keywords: Signal denoising, SURF threshold method, wavelet analysis.

\section{INTRODUCTION}

Wavelet analysis plays a very important role in the signal processing. The so-called wavelet transform is that the data process or data series is converted into the cardinal series with some special functions at the base to find its similar spectrum characteristic in order to achieve data processing. Although Mallat wavelet transform is high speed and efficiency in signal processing. Mallat transform method is not suitable for the more complex electromagnetic environment and more quality demanding of the signal. This paper introduces SURF threshold on the basis of Mallat transform, which has a good effect on low frequency signal processing and smooth the signal while reducing concomitant oscillation.

The traditional methods of signal processing usually use the Fourier transform method. Fourier transform is able to meet certain condition's function expressed as trigonometric functions (sine and / or cosine function) or a linear combination of their integral. Fourier transform has a variety of different variations in different areas of research, such as the continuous Fourier transform and the discrete Fourier transform. This method is a kind of application of a wide range of analytical tools, it is a kind of from time domain to frequency domain mutual change means, it will signal is divided into different frequency sine wave superposition. This transformation method two directions have an unlimited extension of the sinusoidal wave as orthogonal basis function, the Fourier transform analysis function of spectrum, finally describes the overall signal time spectrum characteristics, able to describe the characteristics of a stable signal. But because of the diversity and randomness of electromagnetic environment constantly enhanced, the signal quality requirements also gradually improve, but at this time the traditional Fourier transform method to filter white noise signal, to ensure the stability of the signal and effectiveness.

In order to solve the problems of traditional methods, this paper introduces a SURF threshold, the wavelet filter processing, can effectively filter white noise. Design based on the SURF threshold improved Mallat transformation method for denoizing experiments, the results and with a single Mallat wavelet transform method compared with the results, it is concluded that the improved Mallat wavelet transform method can remove a lot of white noise, signal more smooth, fidelity.

\section{THE SUPERIORITY OF WAVELET TRANSFORM}

The traditional signal analysis methods are based on the Fourier transform, whose transformation and inverse transformation formula as equation (1) and (2).

$$
\begin{aligned}
& F(\omega)=F[f(t)]=\int_{-\infty}^{\infty} f(t) e^{-i \omega t} d t \\
& f(t)=F[F(\omega)]=\frac{1}{2 \pi} \int_{-\infty}^{\infty} F(\omega) e^{i \omega t} d \omega
\end{aligned}
$$

Equations (1) and (2) express the transform properties of signal in the frequency domain and time domain. The idea of Fourier transform in small time domain is: Suppose the nonstationary signals are steady in the window analysis functions $g(t)$ in a very small time interval. The window analysis function is moved [1] to make function $f(t) g(t-\tau)$ 


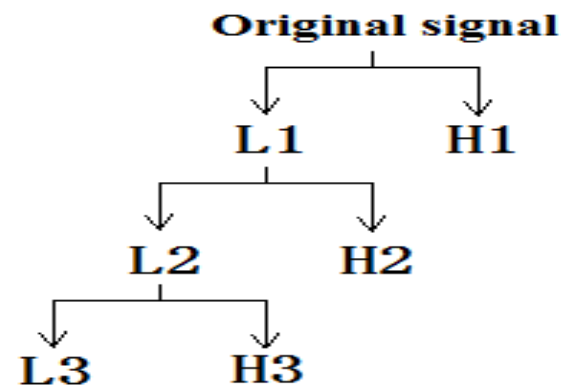

Fig. (1). Tower decomposition of the signal.

steady in different small interval. By this way, the frequency spectrum of the target signal in different time domain. Fourier transform in small time domain: Pseudo-stationary signals are divided into numerous small time-domains, in each domain signal can be seen as a steady signal. Analyzing the signal, the frequency of the signal can be expressed. The formula (3) describes the Fourier transform in small interval $\mathrm{R}$.

$$
F(\omega, t)=\int_{R} f(t) \bar{g}(t-\tau) e^{-i \omega t} d t
$$

$\bar{g}(t)$ is the complex conjugate function of $g(t) . e^{-i \omega t}$ in equation (3) limits the frequency, and $g(t)$ limits time. With the gradual changes of time $\tau$, the Time Window determined by $g(t)$ is moving along the time axis which analyze $f(t)$ in the small interval. The expansion of signal $F(\omega, t)$ can express the status in time interval $[\tau-\sigma, \tau+\sigma]$ and frequency interval $[\omega-\varepsilon, \omega+\varepsilon]$. In order to get better resolution, $\sigma$ and $\varepsilon$ should become infinitesimal. According to Heisenberg uncertainty principle, we know $\varepsilon \sigma \geq \frac{1}{2}$, so $\varepsilon$ and $\sigma$ cannot infinitesimal at the same time, which cause Fourier transform has poor process ability to non-stationary signal.

French engineer J.Morlet presented the concept of wavelet transform in 1984. As a local transformation of time and frequency, it has important meaning for the various signal processing

Assume $\psi(t) \in L^{1}(R) \bigcap L^{2}(R)$ and $\int_{-\infty}^{\infty} \psi(t) d t=0$, then $\psi(t)$ is the basic wavelet. $a$ is the scale parameter and $b$ is the time parameter. The continuous wavelet function is expressed as

$\psi_{a, b}(t)=\frac{1}{\sqrt{|a|}} \psi\left(\frac{t-b}{a}\right)$

Suppose $x(t) \in L^{2}(R)$, and $\psi(t)$ is basic wavelet. The continuous transform of $x(t)$ is
$W T_{x}(a, \tau)=\left\langle x(t), \psi_{a, b}(t)\right\rangle=\frac{1}{\sqrt{a}} \int_{R} x(t) \psi^{*}\left(\frac{t-\tau}{a}\right) d t$ Comparing to

the Fourier transform, wavelet transform overcomes the shortcoming of the window size does not vary with frequency, and can provide a time-frequency window changing with frequency. Therefore, wavelet transform is the ideal tool for frequency analysis for signal processing.

\section{MALLAT TRANSFORM}

\subsection{Mallat Wavelet Transforms Analysis}

The wavelet transform denoising techniques with high speed of signal processing can effectively improve the signal quality.

The wavelet is got by the mutual translation of $\varphi(x)$ the scaling function of $L^{2}(R)$ and wavelet function $\psi(x)$. Before the transform, the wavelet need matrix filtering to make it integrated. Then apply the wavelet transform to signal, and the decomposition of wavelet transform is complete after the filtering.

$H_{k}$ and $G_{k}$ are k matrixes $(r \times r)$ of $\mathrm{r}$ wavelet responsible for filtering.

$\Phi(x)=\left\{\varphi_{1}(x), \varphi_{2}(x), \ldots, \varphi_{r}(x)\right\}^{T}$ is the scale function vector of wavelet $\mathrm{r}$.

$$
\Psi(x)=\left\{\psi_{1}(x), \psi_{2}(x), \ldots, \psi_{r}(x)\right\}^{T} \text { is the space wavelet }
$$
function vector of $r$. The wavelet transform process can be described as equation (5) and (6).

$$
\begin{aligned}
& \Phi(x)=2 \sum_{k} H_{k} \Phi(2 x-k) \\
& \Psi(x)=2 \sum_{k} G_{k} \Psi(2 x-k)
\end{aligned}
$$

In Mallat transform, the scaling function can be understood as the low-pass filter coefficient of the decomposition, and the wavelet coefficients can be understood as high-pass filter coefficient of the decomposition.

\subsection{The Image Signal Denoising Model of Mallat Wave- let Transforms}

The wavelet transform can be seen as a limited signal model. For a one-dimensional discrete signal, first layer lowfrequency signal L1 and first layer high-frequency signal H1 can be obtained through a layer of wavelet transform. The first layer low-frequency signal can be decomposed to the second low-frequency signal L2 and the second highfrequency signal H2. L2 can be decomposed to the third lowfrequency signal L3 and the third high-frequency signal H3. This is the process of tower decomposition, as shown in (Fig. 1). 

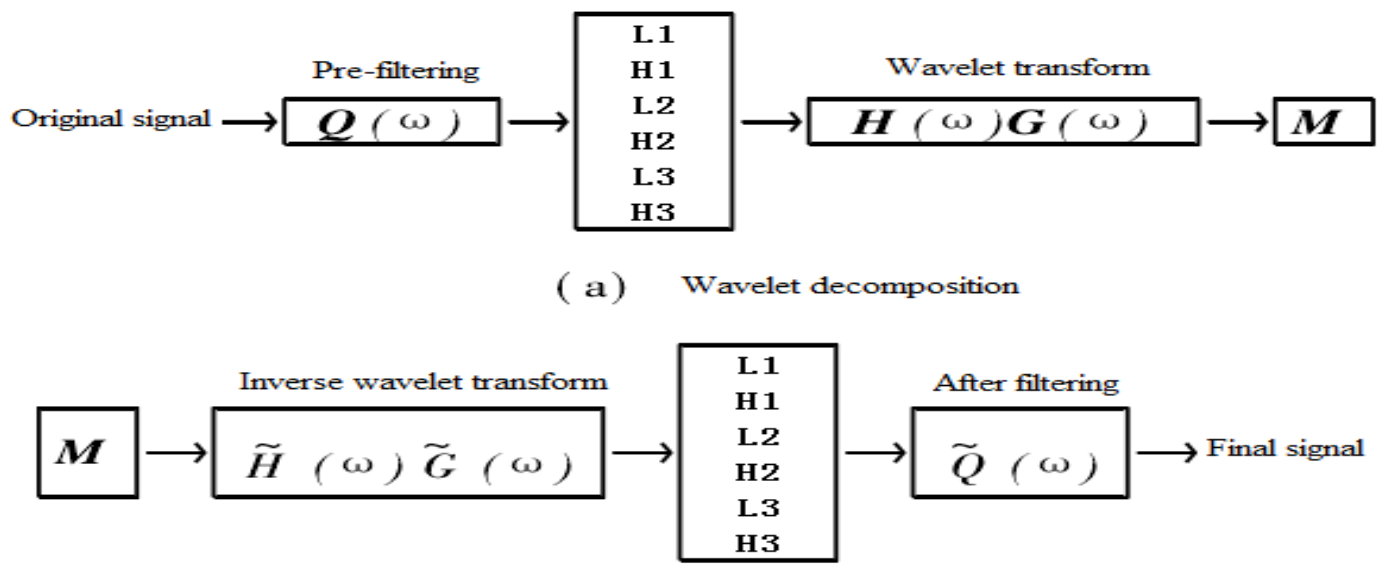

(b) Wavelet reconstruction

Fig. (2). Decomposition and reconstruction of the wavelet.

The process in Fig. (2) is called two layers wavelet decomposition. Wavelet decomposition decomposes only the low-frequency signal. The more detailed the low-frequency signal is decomposed, the more improved the signal resolution is. With the gradual breakdown of the signal, its processing difficulty will increase, and the effect is vaguer.

In the decomposition and reconstruction of the wavelet, the total pixels of the images are constants. $H(\omega)$ and $G(\omega)$ are wavelet filter matrix which also called wavelet filter. $\tilde{Q}(\omega)$ and $Q(\omega)$ are wavelet inverse filter [2].

\section{THE IMPROVED MALLAT WAVELET TRANS- FORM BASED ON SURF THRESHOLD}

The disadvantage of Mallat wavelet transform is that its scope of application is not extensive. It is very effective when the range and the frequency of the signal are known and the frequency bands are separated from each other. The denoising effect is poor to the white noise widespread [3]. In order to solve this problem, we propose using SURF threshold method to improve Mallat wavelet transform.

The actual signal contains a lot of noise, here $N_{i}$ indicates the Gauss white noise, $W_{i}$ represents signal with noise and $S_{i}$ represents the ideal signal (without noise). We get $W_{i}=S_{i}+\varepsilon N_{i}$ where $\varepsilon$ is the control factor if noise intensity obeying $N\left(0, \sigma^{2}\right)$.

The Wavelet differentiated need to determine the SURF threshold before treatment. First we need to estimate the image variance and noise variance. To noise variance $\sigma_{Y}^{2}$

$\sigma_{Y}^{2}(s, j)=\frac{1}{n^{2}(j)} \sum_{i, j=1}^{n(j)} N^{2}(i, j)$
$N(i, j)$ obey Gauss distribution. The image variance is expressed as

$\sigma_{X}(s, j)=\sqrt{\max \left(\sigma_{Y}^{2}(s, j)-\sigma^{2}(s, j), 0\right)}$

From formula (7) and (8), the optimal threshold is determined as:

$$
T(s, j)=\left\{\begin{array}{c}
\sigma^{2}(s, j) / \sigma_{X}(s, j), \sigma_{X}(s, j)>0 \\
\max (|N(s, j)|), \sigma_{X}(s, j) \leq 0
\end{array}\right.
$$

On the foundation of threshold, the level of wavelet decomposition needs to be determined. The higher the level, the more detailed transformation of low-frequency decomposition and the more improved the signal resolution. With the gradual breakdown of the signal, its processing difficulty will increase, and the effect is vaguer $[4,5]$. The level in the paper is three.

$$
\begin{aligned}
& \Phi(x)=2 \sum_{k} \tilde{H}_{k} \Phi(2 x-k) \\
& \Psi(x)=2 \sum_{k} \tilde{G}_{k} \Psi(2 x-k)
\end{aligned}
$$

Use formula (9) and (10) to reconstruct the wavelet. $\tilde{H}_{k}$ and $\tilde{G}_{k}$ are the wavelet inverse filtering matrix. The wavelet denoising is complete after the reconstruction.

The advantage of threshold denoising method is that the noise is completely suppressed, and peak points reflect the characteristics of the original signal are retained [6].

\section{SIMULATION AND ANALYSIS}

The square wave signal with Gaussian noise is selected as test objects for processing, as shown in (Fig. 3). 


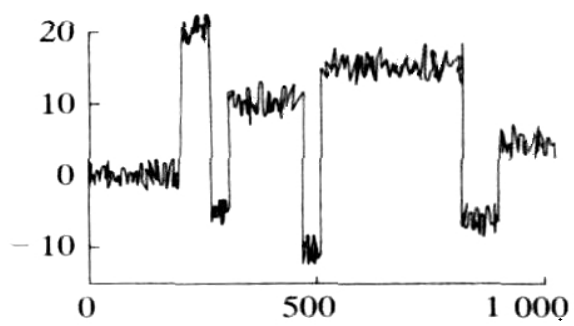

Fig. (3). Noisy signal square wave.

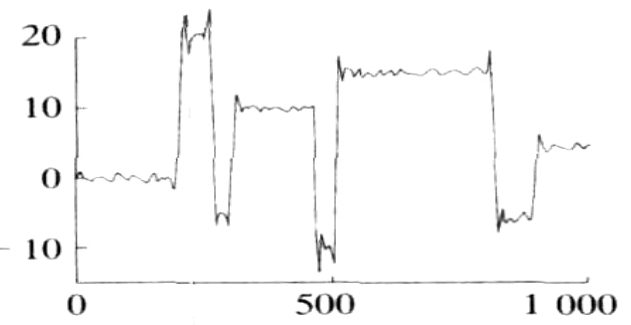

Fig. (4). Transform effect of Mallat.

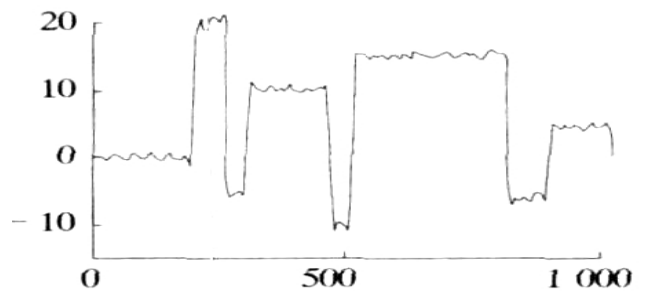

Fig. (5). Transform effect of improved Mallat transform.

The experiments use Matlab 7.0. It compares the denoising effect of Mallat wavelet transform shown in (Fig. 4) and improved method shown in (Fig. 5).

Through contrast of Fig. (4) and Fig. (5), we can obtain that although after Mallat denoising the noise of signal is attenuated, there are still sharp noise points and the phenomenon of white noise is still serious. However, Mallat method is relatively simple and fast, and has good compatibility with Matlab 7.0.

Respectively in order to further verify the effectiveness of the proposed method in this paper, a comparative analysis of the method and the traditional Fourier transform method, in the process of filtered white noise processing efficiency, error rate, and leakage rate, and then through the contrast to validate the effectiveness of the proposed method in this paper.

Through the above three comparative chart available, the method of processing efficiency, error rate, leakage free treatment, are better than the traditional method of Fourier transform. Shows that this method can be timely and effective filtration of white noise, and have high processing precision, and satisfactory results have been achieved.
Based on the filtering effect of SURF threshold, the improved Mallat method well suppress the signal noise and the signal processed is smooth with little additional oscillation [7-12]. It has good signal denoising effect while reserving the processing efficiency and application compatibility.

\section{CONCLUSION}

Analyzing the limitations of Fourier transform in the signal processing, this article reflects the superiority of the wavelet transform, analyzes the principle of Mallat wavelet transform, builds decomposition and reconstruction model of Mallat wavelet, and introduces the SURF threshold to improve Mallat transform method. Finally, comparing the denoising results of single Mallat and improved Mallat, the paper verifies that the improved Mallat method is feasible.

\section{CONFLICT OF INTEREST}

The authors confirm that this article content has no conflict of interest.

\section{ACKNOWLEDGEMENTS}

Declared none. 


\section{REFERENCES}

[1] P. Dong, D. Gao, and Y. Tian, "Improved particle swarm optimization with adaptive inertia weight," Computer Simulation, vol. 29, no. 12 , pp. 283-286, 2012. (in Chinese)

[2] J. Zhang, "Particle swarm optimization using crossover operator", Journal of Convergence Information Technology, vol. 7, no. 4, pp. 287-295, 2012.

[3] X. Fu, J. Liu, L. Sun, and R. Wang, "A new side information estimate based distributed video coding algorithm for wireless multimedia sensor networks", International Journal of Advance Computer Technology, vol. 4, no. 1, pp. 320-326, 2012.

[4] J. Tian, J. Sun, Y. Zhang, N. Ahmad, and B. Zhang, "parallel implementation of compressive sensing based SAR imaging with GPU", Journal of Convergence Information Technology, vol. 6, no. 12, pp. 122-128, 2011.

[5] L. You, G. Zhang, and Z. Fan, "A cryptographic key binding method based on fingerprint features and the threshold scheme", International Journal of Advance Computer Technology, vol. 3, no. 4, pp. 21-31, 2011.

[6] P.E. Abi-char, B. El-Hassan, and A. Mhamed, "An enhanced authenticated key agreement protocol with a neural network-based model for joining-phase in mobile environments", International Journal of Engineering and Innovative Technology, vol. 2, no. 2, pp. 103-112, 2011.

[7] R. Gao, and J. Wu, "An improved ant colony optimization for routing in cognitive radio network", International Journal of Advance Computer Technology, vol. 3, no. 7, pp. 73-81, 2011.

[8] H. Zheng, and Q.F. Luo, "A novel hybrid cuckoo search algorithm based on simplex operator," Journal of Digital Content Technology and its Application, vol. 6, no. 13, p. 45, 2012.

[9] H.Q. Zheng, Y. Zhou, "A discrete cuckoo search algorthm for solving knapsack problems," Advances in Information Science and Service Science, vol. 4, no. 18, p. 331, 2012.

[10] W. Lu, and E.H. Wang, "An improved dv-hop localization algorithm based on the selection of beacon nodes," Journal of Convergence Information Technology, vol. 5, no. 9, p. 157, 2010.

[11] N. Jiang, and X. Xiang, "An iterative boundary node localization algorithm based on DV-hop scheme in WSN," Journal of Convergence Information Technology, vol. 6, no. 7, p. 89, 2011.

[12] C. Tu, "Localization for wireless sensor networks with improved dv-hop and spring-model refinement," Journal of Digital Content Technology and its Application, vol. 6, no. 15, p. 45, 2012.

Received: September 16, 2014

(C) Jiao and Huang; Licensee Bentham Open.

This is an open access article licensed under the terms of the Creative Commons Attribution Non-Commercial License (http://creativecommons.org/licenses/by$\mathrm{nc} / 3.0 /$ ) which permits unrestricted, non-commercial use, distribution and reproduction in any medium, provided the work is properly cited. 